\title{
Zusammenfassung und Fazit
}

Die vorliegende Studie ist im Rahmen des Profilbereiches „Vom Material zur Produktinnovation " der Technischen Universität Darmstadt entstanden und betrachtet die Innovationskette als Ergebnis einer ganzheitlichen Projektdefinition und einer integrierten Lösungsfindung. In diesem Zusammenhang konnte anhand ausgewählter Fallstudien gezeigt werden, dass das Vorhandensein einer soliden Wissensbasis eine fundamentale Voraussetzung für eine erfolgreiche und lückenlose Gewährleistung der Innovationskette darstellt. Somit ist die strategische Grundlagenforschung als unabdingbar für die Produktinnovation anzusehen.

Eine ganzheitliche Projektdefinition und eine integrierte Lösungsfindung implizieren, dass die Entwicklungsprozesse und Wertschöpfungskette nicht als chronologischer Ablauf vorher festgelegter Schritte von der Materialentwicklung zur Produkt-kommerzialisierung betrachtet werden darf. Somit sind abgesehen von klaren und leicht nachvollziehbaren anwendungsspezifischen Voraussetzungen von Anfang an auch nicht-technische Kriterien (bspw. wirtschaftliche Aspekte) zu berücksichtigen. Vor allem ist bei der ganzheitlichen Betrachtung und Bearbeitung der Innovationskette ein Umdenken erforderlich, das alle Schritte bzw. Arbeiten im Rahmen der Wertschöpfungskette am Gesamtoutput misst und beurteilt. Somit sollte gezielt ein disziplinübergreifender Einsatz von Methoden angestrebt werden.

S. Gramlich et al., Vom Material zur Produktinnovation, essentials, https://doi.org/10.1007/978-3-658-20664-2_6 